\title{
Constructing eigenmode excitation spectrum in synthetic photonic lattices using optical heterodyning
}

\author{
A. M. Tikan ${ }^{\mathrm{a}}$, I. D. Vatnik*a, D. V. Churkin ${ }^{\mathrm{b}}$, A. A. Sukhorukov ${ }^{\mathrm{c}}$ \\ ${ }^{\mathrm{a}}$ Novosibirsk State University, Pirogova str. 2, Novosibirsk 630090, Russia; ${ }^{\mathrm{b}}$ Aston Institute of \\ Photonic Technologies, Aston University, Birmingham, B4 7ET, United Kingdom; ${ }^{\mathrm{c} N o n l i n e a r}$ \\ Physics Centre, Research School of Physics and Engineering, The Australian National University, \\ Canberra ACT 2601, Australia
}

\begin{abstract}
A method based on optical heterodyning is proposed for measuring relative optical phases of pulses circulating in a synthetic photonic lattices. The knowledge of the phases can be further used for qualitative reconstruction of an eigenmode excitation spectrum in the synthetic photonic lattice.
\end{abstract}

Keywords: Photonic lattice, eigenmode excitation spectrum, optical heterodyning.

\section{INTRODUCTION}

Light propagation in periodic structures is the object of everyone's attention over the years ${ }^{1}$.This area of research is exploring the dynamics of light, which cannot be observed in a continuous medium. An example of such systems are Bragg grating structures or structures consisting of optical waveguides. The latter are a special class of periodic systems, opening are range of possibilities for controlling light.

Structures consisting of optical waveguides can serve as a platform for observations of a wide variety of physical phenomena. For example, in the work ${ }^{2}$ optical analogue of the Bloch oscillations in the structure of the heat-sensitive waveguide was observed. These oscillations are manifested as a change in the width of the wave packet. Linear law for the propagation constant is achieved by creating a temperature gradient in a direction perpendicular to the light propagation. The propagation of light in waveguide arrays is described by the nonlinear Schrödinger equation. Therefore, optical solitons can be observed in such systems. This phenomenon has been experimentally demonstrated in $1988^{3}$ along with a discrete self-focusing of wave packets. In the paper ${ }^{4}$ authors studied and realized an optical analogue of Rabi oscillations. The oscillations were observed between their modes of photonic lattice. This effect was achieved by periodic modulation of the refractive index in light propagation direction. In addition, in the paper ${ }^{5}$ dynamic localization of the wave packet has been obtained, both in one and in two-dimensional photonic lattices.

Recently more and more attention is given to photonic lattices implemented by means of optical fibers. The main representatives of this class are mesh and synthetic photonic lattices (SPL). The first one is a network of a number of optical couplers, and has some practical limitations. The second one consists of two fiber rings of different lengths, connected through the 50/50 fiber coupler (see Fig.1, left panel). Optical losses are vanished due to optical amplifiers inserted into the system. A sequence of light pulses circulates through the system, with the number of pulse, phase and amplitude of each pulse varying. Phase of the pulses can be altered by means of phase modulator, inserted into one of the loops. It can be shown that pulse evolution in both systems is governed by the same equation set ${ }^{6}$. Though, use of SPL opens new fields for research because of huge experimental possibilities. For example, in the work ${ }^{7}$ an evolution of pulses in a system with the introduction of various kinds of local inhomogeneities was studied. It was shown that the controlled phase shift of each pulse plays a role of quantum potential, while the envelope of the pulse chain plays a role of a wave function of the quantum particle. For example, in the absence of phase shifts train of the pulses circulates in the two rings, with the number of pulses gradually increasing. This can be considered as spreading of the wave function. But if the phase shifts are applied randomly for different pulses, the effect of Anderson localization can be observed in synthetic photonic lattices ${ }^{8,9}$. Indeed, implementing the phase shift for each of the pulses in random fashion is like to obtain an analogue of a random potential in quantum systems. In the absence of phase shifts a pulse chain increases in time from roundtrip to roundtrip, whereas when the random phase shift is applied, the number of pulses in the chain can reach the steady-state level that analogues to the analogue Anderson localization (see Fig. 2).

Nonlinear Optics and its Applications IV, edited by Benjamin J. Eggleton, Neil G. R. Broderick,

Alexander L. Gaeta, Proceedings of SPIE Vol. 9894, 98941L · C 2016 SPIE

CCC code: $0277-786 X / 16 / \$ 18 \cdot$ doi: $10.1117 / 12.2227831$

Proc. of SPIE Vol. $989498941 \mathrm{~L}-1$ 


\section{SINTHETIC PHOTONIC LATTTICE}

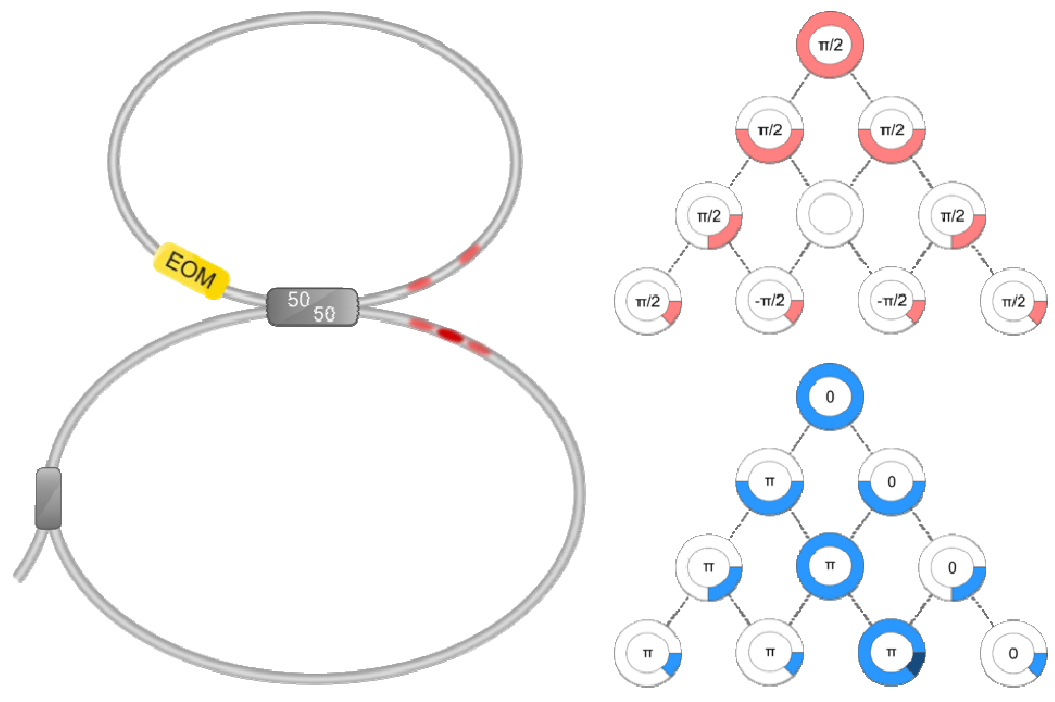

Figure 1. Scheme of a synthetic photonic lattice and basics of an operation.

Fig. 1 shows a scheme of synthetic photonic lattice. The evolution of the pulse in such a system is shown in Fig. 1, right. After the initial pulse is launched into a long loop and makes the first pass, it is divided into two pulses. The pulses have the same intensity, but different relative phases. A pulse propagating in a short loop (upper part of the figure), has an additional phase shift of $\pi / 2$ because of its transition through the coupler. The next pass through the fiber coupler further divides pulses by two, and four pulses (two in each loop) arise because of different lengths of two loops. Phases of the pulses should be $\pi / 2$ and $\pi / 2$ in the small (upper on the Fig.1) loop, 0 and $\pi$ in the large (lower on the Fig.1) loop. The next roundtrip brings constructive and destructive interference into the picture. Indeed, the third roundtrip makes two pulses to be fully overlapped. These two pulses have gone through the same optical path, but have changed the fiber coupler ports different number of times. Therefore the two pulses have phase $\pi / 2$ and $3 \pi / 2$ in the shorter loop, thus leading to destructive interference, while in the larger loop to pulses are with phases $\pi$ and $\pi$, that corresponds to constructive interference. The evolution of this kind corresponds to a ballistic regime of pulse propagation (fig. 2 , left). The typical number of pulses in the pulse chain increases proportionally to the number of rounds.

To observe the effect of Anderson localization one should create a random phase for each pulse in the roundtrip m. In practice, this is carried out by a phase modulator. The resulting pulse evolution strongly deviates from the ballistic regime (fig. 2, right).

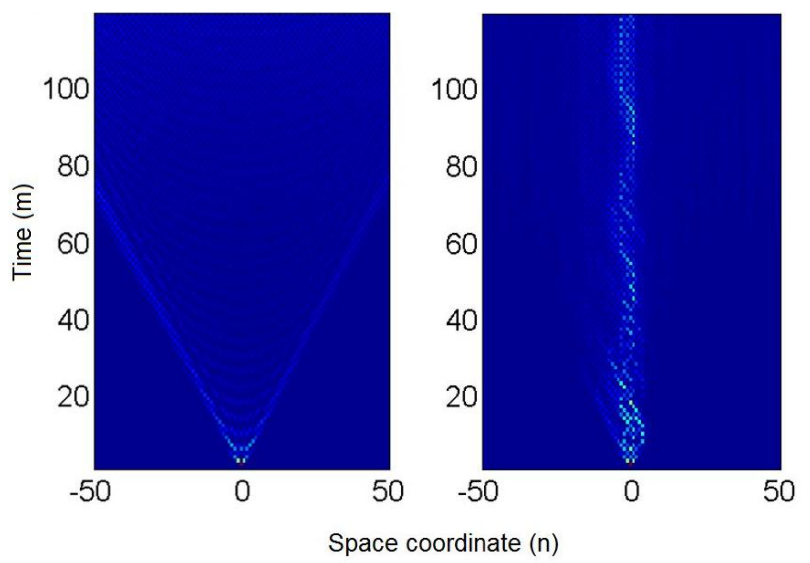

Figure 2.Evolution in a synthetic photonic lattice without optical potential (left) and with randomly distributed phase shifts (right). Data is taken from an experiment. 


\section{EIGENMODE STRUCTURE}

Equation set governing a SPL can be easily obtained ${ }^{6}$ from the basic principles

$$
\left\{\begin{array}{l}
u_{n}^{m+1}=\frac{1}{\sqrt{2}}\left(u_{n+1}^{m}+i v_{n+1}^{m}\right) \\
v_{n}^{m+1}=\frac{e^{i \varphi_{n}}}{\sqrt{2}}\left(v_{n-1}^{m}+i u_{n-1}^{m}\right)
\end{array}\right.
$$

Equations (1) are valid if the coupling ratio is $50 \%$. Here $u$ and $v$ are complex amplitudes of the pulses in long and short loop respectively, situated at the $n$th position in the pulse train at the $m$ th roundtrip.

In case phase is randomly distributed along the coordinate $\mathrm{n}$ and is constant along the coordinate $\mathrm{m}$, function $\varphi$ is, obviously, becomes independent of $\mathrm{m}$ and eigenmodes structure of such system can be found. Eigenmodes of such system can be written in the form of functions, similar to Bloch functions: $u_{n}^{m}=U_{n} \exp (\operatorname{im} \beta)$ - for a long loop, $v_{n}^{m}=V_{n} \exp (\operatorname{im} \beta)$ - for a short loop, where $\beta$-propagation constant.

Then, considering assumption about the eigenmodes form, system of the equations for the two loops system with a random phase shift in each loop and an arbitrary coefficient of the fiber coupler can be written as follows:

$$
\left(\begin{array}{ll}
U U & U V \\
V U & V V
\end{array}\right) *\left(\begin{array}{c}
u_{1}^{m} \\
u_{2}^{m} \\
\vdots \\
u_{N}^{m} \\
v_{1}^{m} \\
v_{2}^{m} \\
\vdots \\
v_{N}^{m}
\end{array}\right)=\left(\begin{array}{c}
u_{1}^{m+1} \\
u_{2}^{m+1} \\
\vdots \\
u_{N}^{m+1} \\
v_{1}^{m+1} \\
v_{2}^{m+1} \\
\vdots \\
v_{N}^{m+1}
\end{array}\right)=\lambda *\left(\begin{array}{c}
u_{1}^{m} \\
u_{2}^{m} \\
\vdots \\
u_{N}^{m} \\
v_{1}^{m} \\
v_{2}^{m} \\
\vdots \\
v_{N}^{m}
\end{array}\right)
$$

here matrices UU, UV, VU, VV - are defined as follows:

$$
\begin{aligned}
& U U=\left(\begin{array}{ccccc}
0 & 0 & 0 & \ldots & \cos (\theta) \exp \left(i \varphi_{1}\right) \\
\cos (\theta) \exp \left(i \varphi_{2}\right) & 0 & \ldots & \ldots & 0 \\
0 & \cos (\theta) \exp \left(i \varphi_{3}\right) & 0 & \cdots & 0 \\
\vdots & \vdots & \ddots & \ddots & \vdots \\
0 & \cdots & 0 & \cos (\theta) \exp \left(i \varphi_{N}\right) & 0
\end{array}\right) \\
& U V=\left(\begin{array}{ccccc}
0 & 0 & 0 & \ldots & i \sin (\theta) \exp \left(i \varphi_{1}\right) \\
i \sin (\theta) \exp \left(i \varphi_{2}\right) & 0 & \ldots & \ldots & 0 \\
0 & i \sin (\theta) \exp \left(i \varphi_{3}\right) & 0 & \cdots & 0 \\
\vdots & \vdots & \ddots & \ddots & \vdots \\
0 & \cdots & 0 & i \sin (\theta) \exp \left(i \varphi_{N}\right) & 0
\end{array}\right) \\
& V U=\left(\begin{array}{ccccc}
0 & i \sin (\theta) \exp \left(i \varphi_{1}\right) & 0 & \cdots & 0 \\
0 & 0 & i \sin (\theta) \exp \left(i \varphi_{2}\right) & \cdots & \vdots \\
\vdots & \vdots & 0 & \ddots & 0 \\
0 & \vdots & \vdots & \ddots & i \sin (\theta) \exp \left(i \varphi_{N-1}\right) \\
i \sin (\theta) \exp \left(i \varphi_{N}\right) & 0 & \cdots & 0 & 0
\end{array}\right) \\
& V V=\left(\begin{array}{ccccc}
0 & \cos (\theta) \exp \left(i \varphi_{1}\right) & 0 & \cdots & 0 \\
0 & 0 & \cos (\theta) \exp \left(i \varphi_{2}\right) & \cdots & \vdots \\
\vdots & \vdots & 0 & \ddots & 0 \\
0 & \vdots & \vdots & \ddots & \cos (\theta) \exp \left(i \varphi_{N-1}\right) \\
\cos (\theta) \exp \left(i \varphi_{N}\right) & 0 & \cdots & 0 & 0
\end{array}\right)
\end{aligned}
$$


where $\theta$ depends on coupler ratio (and is equal to $\pi / 4$ для $50 \%$ coupling ratio).

Thereby, solving the matrix equation (2) eigenvalues and eigenvectors of this system can be found. Propagation constants $\beta_{j}=\log \left(\lambda_{j}\right) / i$ form a dispersion curve. Eigenvalue and corresponding eigenvector form an eigenmode.

To present system (1) as a matrix equation (2) it was necessary to enter the boundary conditions which are considered to be periodic. To demonstrate the structure of eigenmodes, we calculated it in case of no phase shifts applied (Fig.3).
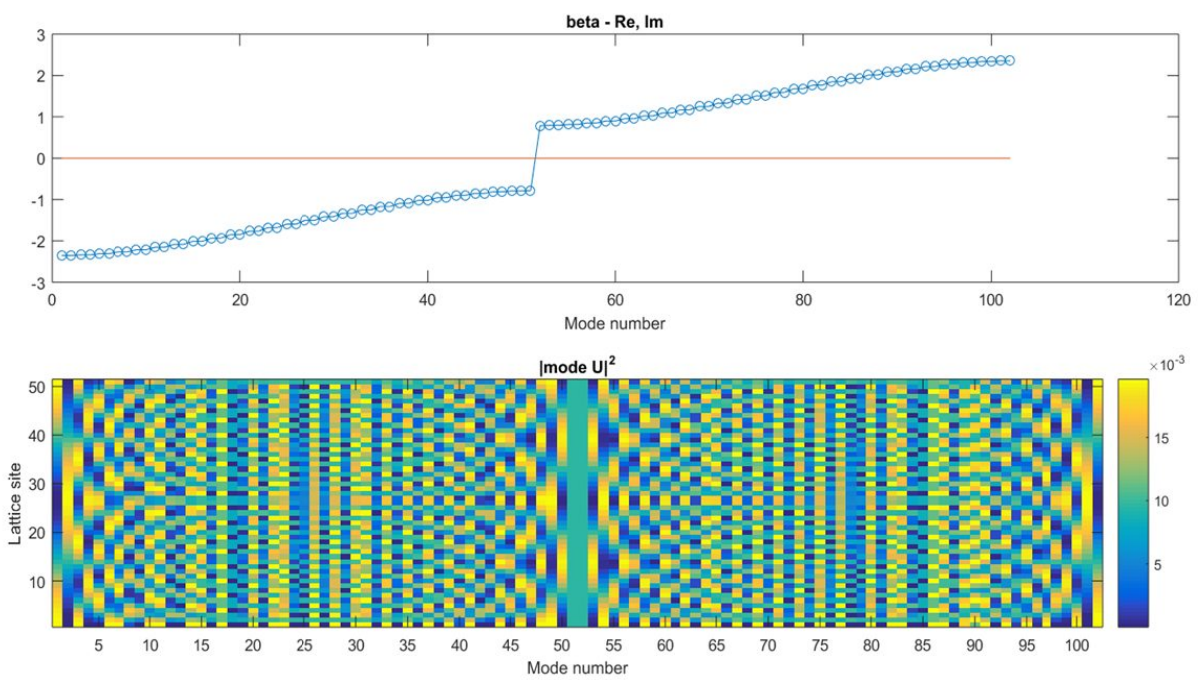

Figure 3.Eigenmode spectrum (propagation constant $\beta$ versus mode number) (upper) and corresponding spatial forms of different modes (lower) for the synthetic photonic lattice without phase shifts.

\section{DERIVING OPTICAL PHASE}

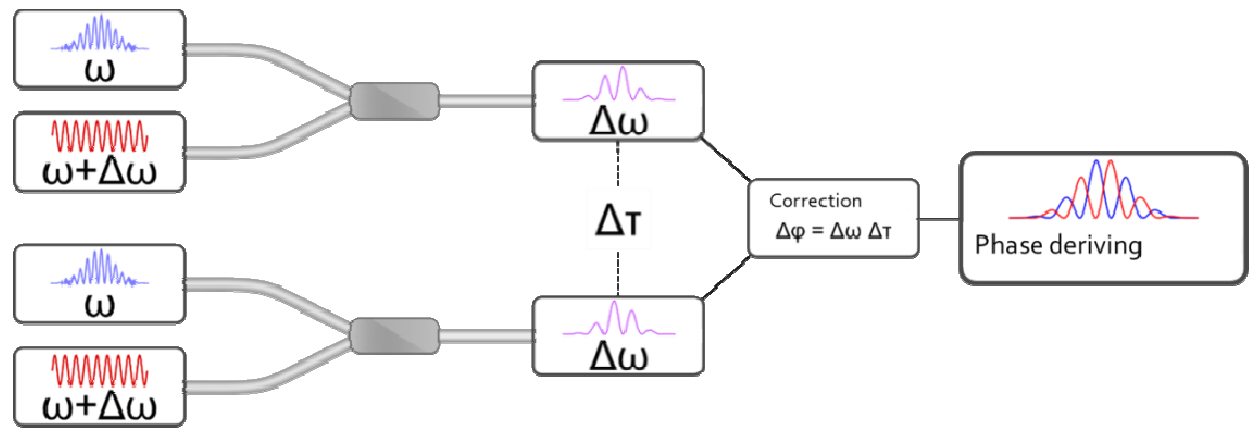

Figure 4. Heterodyning scheme used for deriving the optical pulses phase.

Knowledge of both phase and intensity of the pulse train means the SPL is completely described. So it's of interest to find the relative phases of the pulses in the pulse train.

For phase extraction heterodyne method was proposed. In this method, the pulse chain from the output port coupler from the SPL interferes at the photodiode with a CW signal of local oscillator (LO). Wavelength of local oscillator is chosen to be closed with the frequency of output signal. The result of summation of the signal and LO waves can be found from the formula 


$$
I=\left|U_{S}\right|^{2}+\left|A_{L O}\right|^{2}+2 U_{s} A_{L O}\left(1+\cos \left(\Delta \omega t+\phi_{m}\right)\right)
$$

Here $I$ is resulting intensity at the photodiode, $\mathrm{U}_{\mathrm{s}}$ and $\mathrm{A}_{\mathrm{LO}}$ are fields of the signal and local oscillator correspondingly. As can be seen from the formula (3) the resulting intensity is proportional to the cosine of the difference between the local oscillator frequency and the main signal $\Delta \omega=\omega_{\mathrm{s}}-\omega_{\mathrm{LO}}$. We include an initial phase of the main signal $\phi_{\mathrm{m}}$ in the expression. $\phi_{\mathrm{m}}$ varies from pulse to pulse (for example, upper and lower signal pulses on Fig. 4). In fact, to construct the evolution of the synthetic photonic lattice one should know difference between adjacent pulses. Because different pulses arrive to the photodiode at different times, additional phase $\Delta \omega \Delta \tau$ corresponding to difference in arrival times $\Delta \tau$ appears. Thus, this phase should be subtracted to reveal $\phi_{\mathrm{m}}$. To derive the latter, the following technique is used. We calculate overlap integral, using measured time traces of two adjacent pulses as a function of phase difference $\psi$ :

$$
F(\psi)=\int I_{m 1}(t) I_{m 2}\left(t+\frac{\psi}{\Delta \omega}\right) d t
$$

here $I_{m 1}(t)$ and $I_{m 2}(t)$ are measured intensities for two adjacent output heterodyned pulses. Maximum of $F(\psi)$ can be found numerically and is reached when $\psi$, that determines position of one of the pulses relative to the second one, is equal to $\phi_{\mathrm{m} 1}-\phi_{\mathrm{m} 2}$. Thus, it's possible to derive relative phase between two adjacent pulses in the pulse train, and then caluclate all phases in the pulse train. Together with knowledge of amplitudes of the pulse train, this provides complete information about the evolution of the light in a synthetic photonic lattice.

We simulate an experimental heterodyning technique using equation set (1) and equations $(3,4)$, and construct the matrix of complex pulse amplitudes $\mathrm{U}(\mathrm{m}, \mathrm{n})$. The phase derived by this method exactly reproduced pulse phases derived straightforwardly from the equation set (1).

The technique is due to be proved experimentally though. To make the heterodyning measurements possible, time coherence of the local oscillator should be no less than the time duration $\Delta T_{\text {total }}$ between first and last pulse in the pulse chain. $\Delta T_{\text {total }}$ is proportional to the roundtrip time multiplied by the number of steps over the "time" coordinate $m$. In the synthetic photonic lattices demonstrated up to dates $\Delta T_{\text {total }}$ is about of several milliseconds, thus making strict requirement for the local oscillator bandwidth.

\section{RECONSTRUCTING EIGENMODE EXCITATION SPECTRUM}

Knowledge of both phase and intensity of the pulse train means the SPL becomes completely described. We have found an easy way to qualitatively reconstruct the eigenmode excitation spectrum using this knowledge.

After constructing a matrix of complex pulse amplitudes $\mathrm{U}(\mathrm{m}, \mathrm{n})$ by means of optical heterodyning discussed above, one can calculate the Fourier transform $\widetilde{U}(\beta, n)$ over the "time" coordinate $m$. According to definition of an eigenmode, the Fourier transform is finding all the eigenmodes contributing to the evolution at the $n$th slot. Averaging $\langle\widetilde{U}(\beta, n)\rangle_{\mathrm{n}}$ over the all slots summarizes all eigenmodes involved in the particular realization of a synthetic photonic lattice. We have chosen the SPL with randomly distributed phase shifts as a testbed, i.e. in case when Anderson localization is realized. Calculated intensity of the image $\left\langle|\widetilde{U}(\beta, n)|^{2}\right\rangle_{n}$ indeed has the form similar to the eigenmode excitation spectrum derived analytically using methods described in section 3 (see Fig. 5). Similarity is preserved for different levels of disorder (see Fig. 6). The structure of spectrum with the maxima at the edge of the bandgap, typical for localization processes, is directly reproduced in Fourier transform calculated from the complex amplitude $\mathrm{U}(\mathrm{m}, \mathrm{n})$.
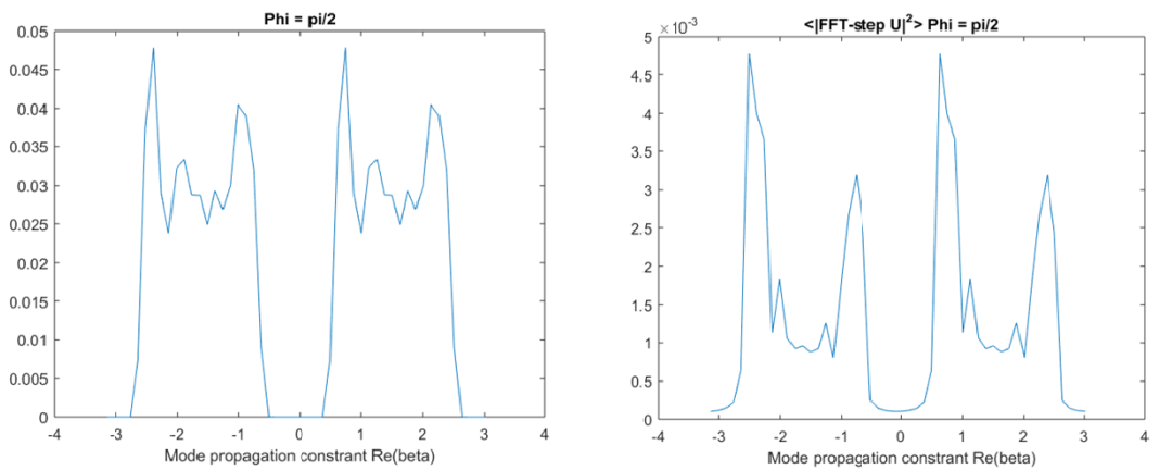

Figure 5. Comparing mode excitation spectrum (left) and result of Fourier transform of complex pulse amplitude over $\mathrm{m}$ (right) with random distribution of potential with value of $\pi / 2$. 

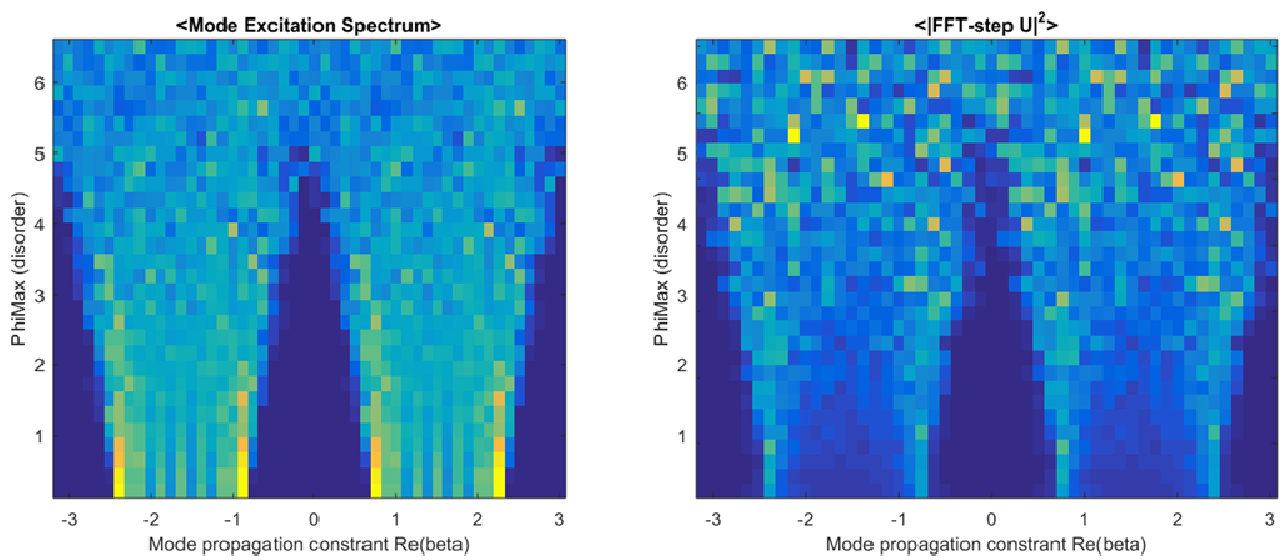

Figure 6. Comparison of mode excitation spectrum (left) and result of Fourier transform of evolution of the SPL (right) for different levels of potential disorder.

\section{CONCLUSION}

We propose a technique of measuring relative optical phases of pulses circulating in synthetic photonic lattices. The technique is based on optical heterodyning and makes it possible to obtain complete knowledge about light evolution in the synthetic photonic lattices. One of the sequences is the possibility of reconstructing the eigenmode excitation spectrum, using measurements of output pulse train phases and intensities only. We demonstrate that the Fourier transform intensity of the complex amplitude over roundtrip number $m$ has a shape similar to that of eigenmode excitation spectrum.

\section{REFERENCES}

[1] Joannopoulos, J. D., Johnson, S. G., Winn, J. N.., Meade, R. D., Photonic Crystals: Molding the Flow of Light, 2nd ed. (2008).

[2] Pertsch, T., Dannberg, P., Elflein, W., Bruer, A.., Lederer, F., "Optical Bloch Oscillations in Temperature Tuned Waveguide Arrays,” Rev. Lett. 83, 4752 (1999).

[3] Christodoulides, D. N.., Joseph, R. I., "Discrete self-focusing in nonlinear arrays of coupled waveguides," Opt. Lett. 13, 794 (1988).

[4] Shandarova, K., Rüter, C. E., Kip, D., Makris, K. G., Christodoulides, D. N., Peleg, O.., Segev, M., "Experimental Observation of Rabi Oscillations in Photonic Lattices," Phys. Rev. Lett. 102(12), 123905 (2009).

[5] Schwartz, T., Bartal, G., Fishman, S.., Segev, M., "Transport and Anderson localization in disordered twodimensional photonic lattices," Nature 446(7131), 52-55 (2007).

[6] Regensburger, A., Miri, M., Bersch, C., Näger, J., Onishchukov, G., Christodoulides, D. N.., Peschel, U., "Observation of Defect States in PT-Symmetric Optical Lattices," Optics; Quantum Physics, Arxiv Prepr. (2013).

[7] Regensburger, A., Bersch, C., Hinrichs, B., Onishchukov, G., Schreiber, A., Silberhorn, C.., Peschel, U., "Photon Propagation in a Discrete Fiber Network: An Interplay of Coherence and Losses,” Phys. Rev. Lett. 107(23), 233902 (2011).

[8] Schreiber, A., Cassemiro, K. N., Potoček, V., Gábris, A., Jex, I.., Silberhorn, C., "Decoherence and Disorder in Quantum Walks: From Ballistic Spread to Localization,” Phys. Rev. Lett. 106(18), 180403 (2011).

[9] Vatnik, I., Tikan, A. M., Churkin, D. V.., Sukhorukov, A. A., "Anderson Localization in Synthetic Photonic Lattices," CLEO 2015, FTh3D.1, OSA, Washington, D.C. (2015). 